Journal of

Back and

Musculoskeletal

Rehabilitation

Journal of Back and Musculoskeletal Rehabilitation 8 (1997) 83-85

Introduction

\title{
Technological advance amidst humanistic decline: Ignoring muscle evaluation and treatment in the modern age of medicine
}

\begin{abstract}
'Medicine may be the only discipline that lacks a comprehensive Theory... A Theory must account for all the factors that predispose to, initiate, or sustain a disease'.
\end{abstract}

Weiner, H. 1977 [1]

As a group, pain medicine clinicians often fail to help back pain patients. The failures are constant reminders that we lack a cohesive theory of the etiology and treatment of clinical pain. Until recently, we each parochially practiced our own specialized approach in a vacuum, without the need to justify why we did things our way.

The current reimbursement crisis is changing things. The stampede is on for outcomes assessment to establish the value (outcome/cost) of interventions. But how you arrive at 'outcome' is crucial. The clinicians' ability to practice the way they want will hinge on showing that their 'customers' accept the value of the service.

There are several outcomes measures in use today. But so far none is a comprehensive, globally accepted instrument. The American Academy of Pain Medicine's Uniform Outcomes Measures Committee (UOMC), of which I am Chairman, has been working on a standardized outcomes assessment tool for 6 years. We are now field testing a prototype. The lead article summarizes the UOMCs work.

The Committee's work is more difficult because pain medicine is a multidisciplinary field. Team members often hold diametrically opposing views on the etiology and treatment of similar symptoms.

Opposing views were never more clear than in the creation of this edition. Some articles were close to heresy, even stupidity in the opinions of some reviewers. To encompass this broad range of opinion, we chose a point-counterpoint format. Throughout, we incorporated the idea that muscles are often overlooked or underemphasized as a cause of back pain.

This issue is a tribute to Hans Kraus, MD who died in March of 1996 at the age of 90 . He led an extraordinary life: world-renowned physician, author, and mountain climber. Kraus provided the spark to President Eisenhower that ignited the President's Council on Physical Fitness in 1955. He reported on the failure of $50 \%$ of American children vs. $4 \%$ of European children to pass a basic test of postural trunk muscle strength and flexibility.

He returned to the White House in 1963 when Eugene Cohen, MD, President Kennedy's endocrinologist, requested Kraus to consult in the treatment of Kennedy's back pain. Kennedy had been getting up to six 'trigger point injections' a day without any lasting relief of his pain. Kennedy had two failed laminectomies and had suffered an acute strain when he planted a tree with Prime Minister Trudeau in Canada in early 1963. 
Kraus diagnosed Kennedy as having spasm, weakness, stiffness and trigger points. He first treated the spasm, not with injections but with electrical stimulation, ethyl chloride spray, and gentle limbering. Kraus took charge of all the President's functional muscular diagnoses, resulting in almost total relief of pain [2]. John and Robert Kennedy saw that an organ system had been overlooked in the conceptual approach to back pain. They planned to create a National Muscle Institute before they were assassinated.

In the second article of this issue, Kraus and Marcus present Kraus' pioneering work in creating an exercise program to relieve back pain. Over 4.5 years beginning in 1945, Kraus worked with 3700 patients at Columbia University Medical School. The exercises formulated at Columbia were the basis for the YMCAs 'Y's Way to a Healthy Back' Program. It has been given to over 300000 back pain sufferers with over $80 \%$ reporting full recovery.

In the third article, Wyszynski presents how we incorporated these exercises into the New York Pain Treatment Program, a multidisciplinary pain treatment program. His contributions are personally satisfying to me; he joined our program planning to drop our Kraus-derived methods. Now he supports the Kraus systematic evaluation and treatment protocol.

One of the difficulties in conceptualizing modern back pain is the seductiveness of modern diagnostic imaging. Anatomical variations in spine structure may mistakenly be considered 'abnormal', not simply anatomical variations and the effects of age. This can lead to treatment for these 'abnormalities'. In the forth paper, Zimmerman presents important data that should help us use the newer imaging technologies more thoughtfully and cost-effectively.

The lack of a standard way to evaluate and treat pain of muscular origin has caused overemphasis on findings in the neuraxis and skeleton as the sole pain generators. The success rate of epidural steroids would rise with the inclusion of other putative factors in the etiology of low back pain. The goal here is better case selection.

In the fifth paper, Abrams reviews the literature and use of epidural steroid injections for the treatment of low back pain. His conclusions may help us focus our selection criterion, leading to a greater success rate and higher consumer value.

Determining pain and tenderness during a physical examination is full of subjectivity and unreliability. In the sixth article, Fisher presents an instrument that provides a measure of objectivity in the assessment of muscle tenderness. The article and the counter point discuss muscle pain and tenderness nomenclature and reinforce the need for generally accepted standardized definitions of clinical muscle pain.

In reviewing predisposing factors for the occurrence of back pain, endocrinological deficiencies are not generally considered. Sonkin presents data suggesting functional hypothyroidism may exist secondary to end organ lack of response without feedback to the pituitary hypothalamic axis. The patient thus presents a normal thyroid profile and TSH.

He presents his experience with administration of exogenous thyroid replacement resulting in lowered serum cholesterol and decrease in muscle tenderness as well as decrease in all of the typical signs and symptoms of hypothyroidism. We have treated a number of patients at my center with Sonkin's protocol with positive results. This subject deserves further study.

Finally, the commentary by Starr presents a physician's personal case study of differing responses to two very different approaches - both called trigger point treatment.

Despite the enormous differences of opinion among participants, several unifying themes emerged as we prepared this issue:

- For now, double blind studies should not be the only criteria we use to decide how we treat patients. We need generally accepted definitions, inclusion and exclusion criterion in similar studies, and uniform outcome measurement. Until then, it will continue to be tough to move our understanding of effective pain treatment ahead quickly.

- Our approaches to back pain should adhere to principles of common sense and respect for the findings of past clinicians. Just as cardiac medications derived from digitalis have cont- 
inued to be standard treatment in spite of newer drugs, we need to use what has worked over time.

- Without the inclusion of muscle as a standard part of the physical examination, we will continue to exclude a major cause of back pain and perpetuate ill conceived and ill advised evaluations and treatments.

Seeing the need to educate the lay and professional communities about evaluating and treating pain, I started the non-profit International Foundation for Pain Relief in 1994. It will fund studies and disseminate findings on cost-effective approaches to common pain problems. IFPR welcomes contributions and suggestions for future research studies.

N.J. Marcus, $M D$

\section{References}

[1] Weiner H. Psychobiology and human disease. New York: Elsevier North Holland, 1977, p. xi.

[2] Reeves R. President Kennedy. Profile of power. New York: Simon and Schuster, 1993, pp. 242, 243, 273. 